\title{
Los costos ocultos de la Cartografía*
}

\author{
José María Lazo Pritsch ${ }^{* *}$
}

Recibido el 14 de abril de 2015; aceptado el 16 de agosto de 2015

\begin{abstract}
Appropriate and high quality Geographic Information is becoming increasingly important. It is essential in all country's activities, and the Spatial Data Infrastructure plays an essential role when sharing the information. We must generate and maintain it, but we also need to finance it. Usually a cost-benefit analysis in performed in order to make decisions regarding the investment in projects on Geographical Information. However, there are some "hidden" costs in the generation of geographic data that are generally not considered in these analyzes, but should be. These are called "Opportunity Cost" and "Cost of Poor Quality". The opportunity cost is the value of the unrealized best option. If it had not been decided to make the photogrammetric flight in 1966-1967, then, what would have been the impact and the opportunity cost? On the other hand, the costs of poor quality correspond to external faults, those errors incurred by the producer because the external client is being provided with unacceptable products or services. Mistakes in the information provided by the state to a construction company, eventually costed around 15 million dollars, which could have been avoided if quality mapping had been provided.

Both costs can be useful to help making investment decisions.

These hidden costs, can they, alone or together with a cost-benefit analysis, be actually used to make investment decisions in Geographical Information?

Can these costs, both of poor quality as well as the opportunity cost, be such as to influence that decision?
\end{abstract}

Key words: Opportunity cost, cost of poor quality.

\section{Resumen}

La Información Geográfica de calidad y oportuna cada vez toma mayor importancia. Es imprescindible en todas las actividades de un país, jugando un rol esencial la

* Esta es una versión revisada y extendida del trabajo del mismo título presentado en las II Jornadas de Cartografía, 30-31 de octubre de 2014, Montevideo, Uruguay.

** Montevideo, Uruguay, correo electrónico: jlazo007@gmail.com 
Infraestructura de Datos Espaciales (IDE) al compartir esa información. Hay que generarla y mantenerla, pero también hay que financiarla; es común que se utilice un análisis Costo-Beneficio para tomar decisiones con respecto a la realización de proyectos de inversión en Información Geográfica, sin embargo hay algunos costos "ocultos" en la generación de los datos geográficos que generalmente no se tienen en cuenta en estos análisis, pero deberían serlos. Ellos son los llamados "Costo de Oportunidad" y el "Costo de la Mala Calidad". El Costo de Oportunidad es el Valor de la mejor opción no realizada. Si no se hubiera decidido hacer el vuelo fotogramétrico de 1966-1967, entonces, ¿cuál hubiera sido el impacto y cuánto el costo de oportunidad? En cambio los costos de la mala calidad correspondiente a fallos externos, son todos aquellos errores en que incurre el productor porque al cliente externo se le suministran productos o servicios inaceptables. Por errores en planos provistos por el Estado a una empresa constructora, hubo que pagar a esta última alrededor de 15 millones de dólares, que se podría haber evitado si se hubiera dispuesto de cartografía de calidad y oportuna.

Ambos costos pueden ser de utilidad para ayudar a la toma decisiones de inversión.

¿Estos costos ocultos, aislada o conjuntamente con un análisis Costo-Beneficio, podrán ser utilizados realmente para tomar decisiones de inversión en Información Geográfica?

¿Podrán estos costos, tanto el de la mala calidad así como el costo de oportunidad, ser de tal magnitud como para influir en esa decisión?

Palabras clave: costo de oportunidad, costo de la mala calidad.

\section{Resumo}

A Informação Geográfica apropriada e de qualidade ganha cada vez mais importância. É imprescindível em todas as atividades de um país, levando-se em conta que as Infraestrutura de Dados Espaciais (IDE) tem um papel essencial no compartilhamento dessa informação. Para gerá-la e mantê-la, e também financiá-la são comuns análises do custo-benefício para tomar decisões que dizem respeito ao investimento em projetos de Informação Geográfica. No entanto, existem alguns custos "ocultos" na obtenção de dados geográficos que geralmente não são levados em conta nesta análise, quando deveriam ser. Eles são chamados Custos de Oportunidade e Custos de Baixa Qualidade. O custo de oportunidade é o valor da melhor opção não realizada. Se não houvesse sido decidido por fazer o vôo fotogramétrico de 1966-1967 qual seria o impacto e o custo de oportunidade? Por outro lado, os custos de baixa qualidade correspondem a falhas externas, sendo todos os erros que incorrem ao produtor porque o cliente externo fornece produtos ou serviços inaceitáveis. Por conta de erros nos planos entregues pelo Estado a uma construtora, este teve que pagar cerca de 15 milhões de dólares, o que poderia ter sido evitado se 
tivessem usado de cartografia apropriada e de qualidade. Ambos os custos podem ser utilizados para ajudar na tomada de investimento. Estes custos ocultos ou juntamente a análise custo-benefício, poderão ser utilizados para tomar decisões de investimento em Informação Geográfica? Poderão eles, tanto o de baixa qualidade quanto o de oportunidade, serem de escala tal para influir nessa decisão?

Palavras-chave: custo de oportunidade, custo de baixa qualidadde.

\section{Información Geográfica, Proceso de Producción e Infraestructura de Datos Espaciales}

La cartografía y su expresión, el mapa, ha sido la respuesta a la necesidad de disponer de un modelo de la realidad de lo que ocurre en el territorio, teniendo como finalidad aportar Información Geográfica (IG) de ayuda para la toma de decisiones. "Cada vez se reconoce más, tanto en gobiernos como en el sector privado, que comprender la ubicación y lugar es un componente fundamental para esa toma de decisiones efectiva", Carpenters y Snell (2013). Cualquier actividad humana u objeto geográfico puede representarse en un mapa. Un porcentaje muy alto de los datos generados diariamente en Internet, que se estiman en alrededor de 2.5 trillones de bytes de datos $(2.5 \times 1,018)$, IBM $(2014)$, contienen referencia de localización geográfica. Es tan vital la IG que se dice que es "la infraestructura de las infraestructuras", Canas (2001), porque con ella se diseñan, planifican, construyen y gestionan otras grandes infraestructuras, como carreteras y puentes, vías férreas, represas y presas, saneamiento, entre otras obras. Se afirma que "la información geográfica es crítica para promover el desarrollo económico, mejorar nuestra gestión de los recursos naturales y proteger el medio ambiente", Clinton (1994). Los análisis de impacto realizados corroboran que la cadena de servicios de valor agregado que se produce alrededor del dato geográfico, que normalmente viene dado por su costo de generación y actualización, aumenta sustancialmente el beneficio económico del mismo. Ni que hablar sobre el valor del dato cuando éste es ofrecido a través de las Infraestructuras de Datos Espaciales (IDE). Éstas tienen el componente geográfico (datos, metadatos y servicios), un componente tecnológico (arquitectura cliente-servidor, normas y estándares), un componente social (productores, desarrolladores, usuarios, etc.) y un componente político, que se muestran en la Figura 1.

El componente geográfico, refiriéndose a los datos, está en la base de la pirámide de componentes y sin él es imposible la IDE. Está constituido por los datos fundamentales (o de referencia) y los temáticos. Los fundamentales son aquellos que sirven de base para construir o referenciar los otros datos, los temáticos, y se constituyen en la cartografía básica de un país. Habitualmente se consideran en este tipo el control geodésico, relieve, red de transporte, hidrografía, unidades políticoadministrativas, suelos, catastro, los nombres geográficos y las ortofotos. Como 
datos temáticos, dentro de una lista larga, podemos mencionar los de geología, medioambientales, forestales, demografía y clima.

La generación, el mantenimiento y/o el acrecentamiento de su calidad y actualidad y en consecuencia su valor, son esenciales para las IDE.

El proceso cartográfico para la generación y mantenimiento de los datos fundamentales, es decir de su base de datos, consta de dos grandes etapas: la adquisición o recolección y la gestión o procesamiento de datos. La adquisición de datos es el proceso mediante el cual se obtienen los objetos y su localización a partir de imágenes satélites, fotos aéreas, LIDAR, etc., con el auxilio de puntos de control obtenidos de la red de estaciones GNSS. En tanto que la gestión de datos son todos los procesos de generalización, edición, generación de MDT, ortofotos, control de calidad, etc. (véase Figura 2).

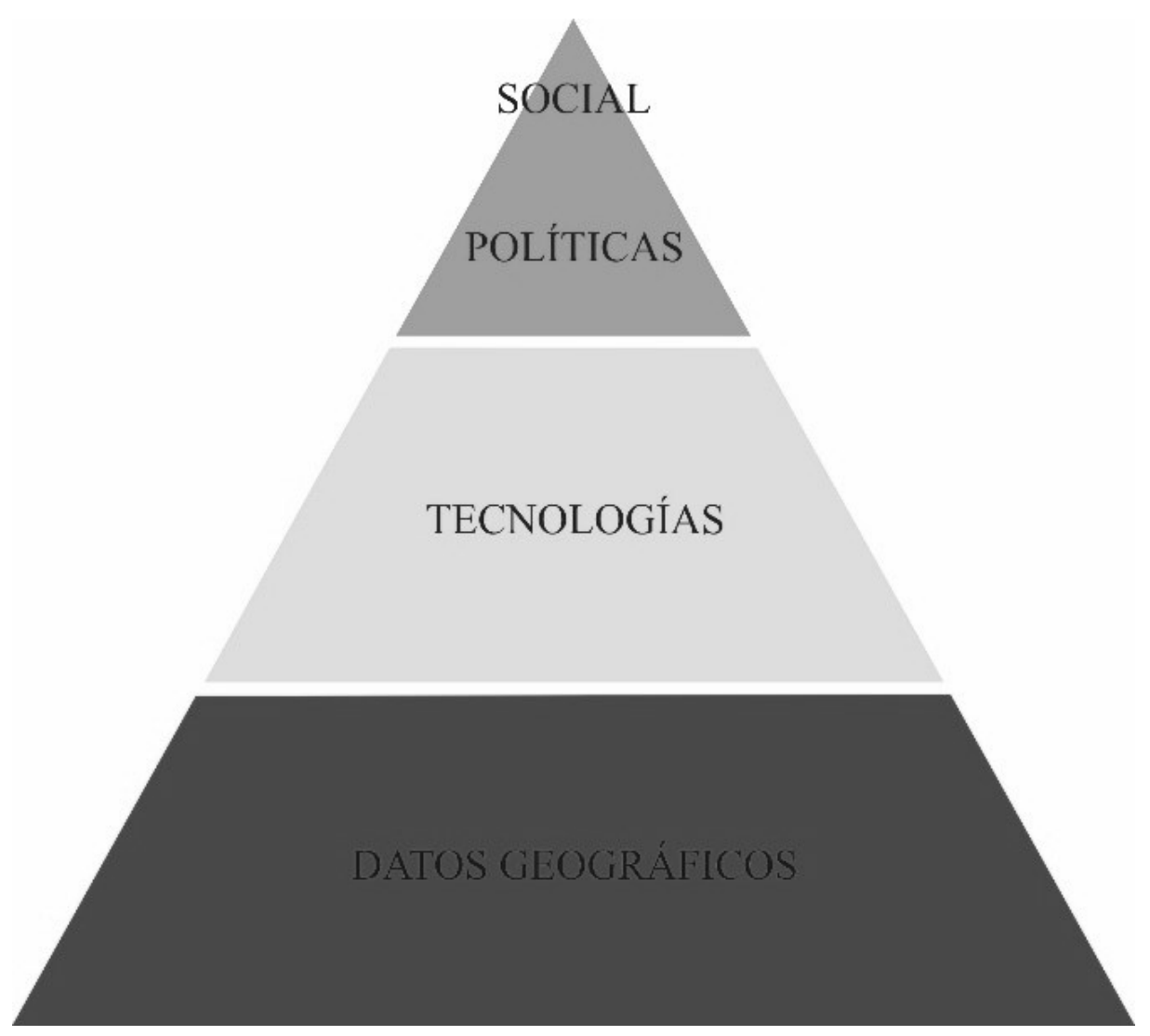

Figura 1. Componentes de la IDE.

Fuente: Elaboración propia. 


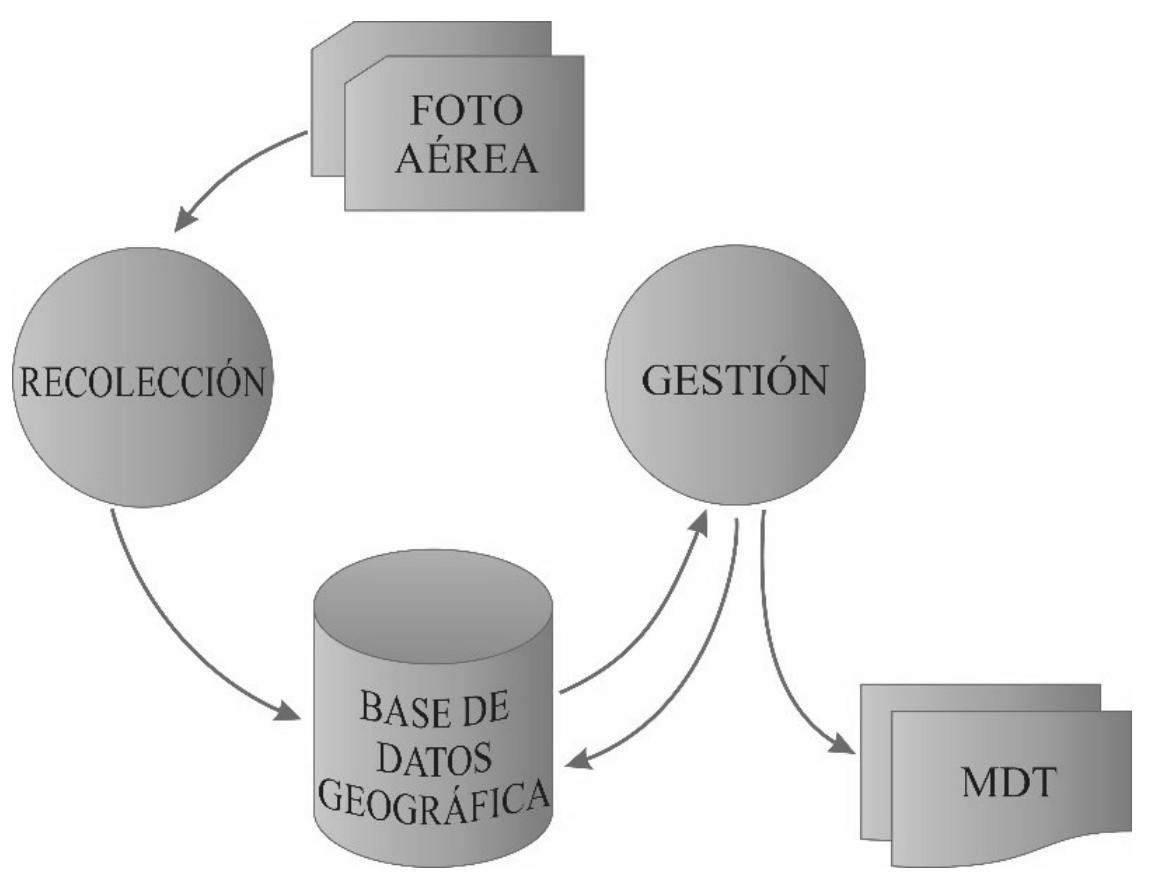

Figura 2. Proceso cartográfico.

Fuente: Elaboración propia.

El mantenimiento de los datos se realiza para tener la información geográfica actualizada y cada vez con mayor calidad y por lo tanto con mayor valor. La frecuencia con que se debe hacer este proceso llamado ciclo de actualización depende del dinamismo del territorio en cuestión y la tecnología, pudiendo ser comunes ciclos de 3 a 10 años. Se ha observado que dicho ciclo parece haber evolucionado siguiendo en general una curva logarítmica donde, por ejemplo, en la década de los veinte era de unos 30-40 años, en la década de los setenta de 10 años y hoy de hasta 3 años y menos.

En países con recursos cartográficos e IDEs menos desarrollados, habrá una mejor comprensión del papel crucial de los datos de alta calidad para apoyar el desarrollo económico y social. Es probable que se dediquen porciones relativamente más altas de capital nacional a programas de captura y mantenimiento, a medida que hay mayor conciencia y comprensión del valor de contar con información geoespacial precisa y con mantenimiento, para otras prioridades políticas (Carpenters y Snell, 2013).

Cada vez surgen nuevas tecnologías para la recolección de datos así como para la gestión y que aportan mejores datos, de mayor calidad y más accesibles, es decir aumentan su valor. 


\section{Calidad y mala calidad}

Por consiguiente el tema de la calidad de los datos es de enorme importancia. La norma ISO 8402 (1986) define la calidad como "el conjunto de características de una entidad le confieren la aptitud para satisfacer las necesidades establecidas e implícitas", Wikipedia (1).

La calidad de los datos así como de las bases de datos geográficas digitales, están guiadas por los estándares, en especial las normas correspondientes al TC 211 ISO 19113, 19114, 19135 y 19138. Los elementos generales de calidad (no cuantitativos) tienen que ver con el uso, propósito y linaje de los datos. En cambio los propios de la calidad (cuantitativos) son la exactitud posicional, temática y temporal así como la consistencia lógica y compleción.

Estos elementos de la calidad deben estar descriptos en los metadatos. Otro estándar de aptitud de uso de los datos es por ejemplo el NSSDA (FGDC, 1998).

La mejora de la calidad del mapa no solo tiene beneficios obvios para el usuario en términos de exactitud y precisión, sino que también desempeña un papel importante en la economía de la producción cartográfica al afectar los costos de producción y la rentabilidad.

Hay un ciclo virtuoso que se da: costo $\rightarrow$ calidad $\rightarrow$ beneficios (véase Figura 3).

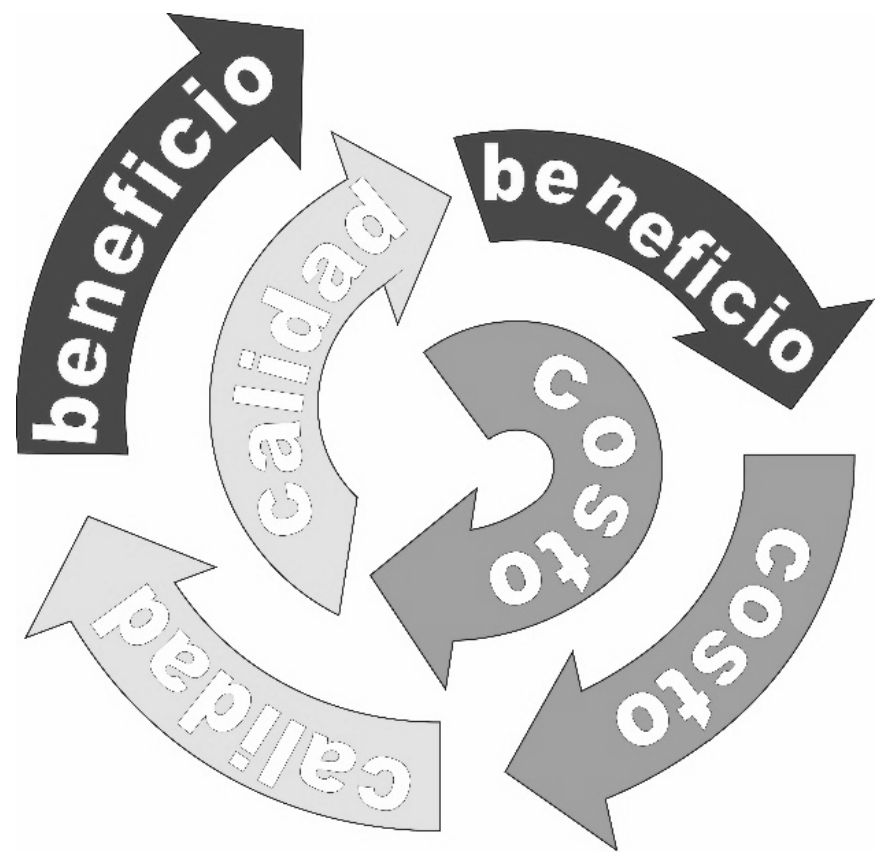

Figura 3. Ciclo virtuoso del costo, calidad y beneficio.

Fuente: Elaboración propia. 
Pero no hay que olvidar que la calidad tiene costos, éstos son típicamente de: prevención, evaluación, fallos internos y fallos externos. Los costos de prevención son todos los gastos realizados para evitar que se cometan errores. El costo de evaluación es el resultado de presupuestar la producción ya terminada y la auditoría del proceso. Los costos de baja calidad son llamados también de mala calidad (CMC). Según López Fojo (2014), al seguir su enfoque de costo de la baja calidad, define este término como la suma de los costos internos o externos. Estos CMC internos son todos los errores que tiene la empresa y que han sido detectados antes que los bienes o servicios sean aceptados por el cliente. Los CMC externos son todos aquellos errores en que incurre el productor porque al cliente externo se le suministran productos o servicios inaceptables, García y otros (2002). Estos costos de fallas son llamados resultantes y son considerados pérdidas (véase Figura 4).

\section{Los costos ocultos de la Cartografía}

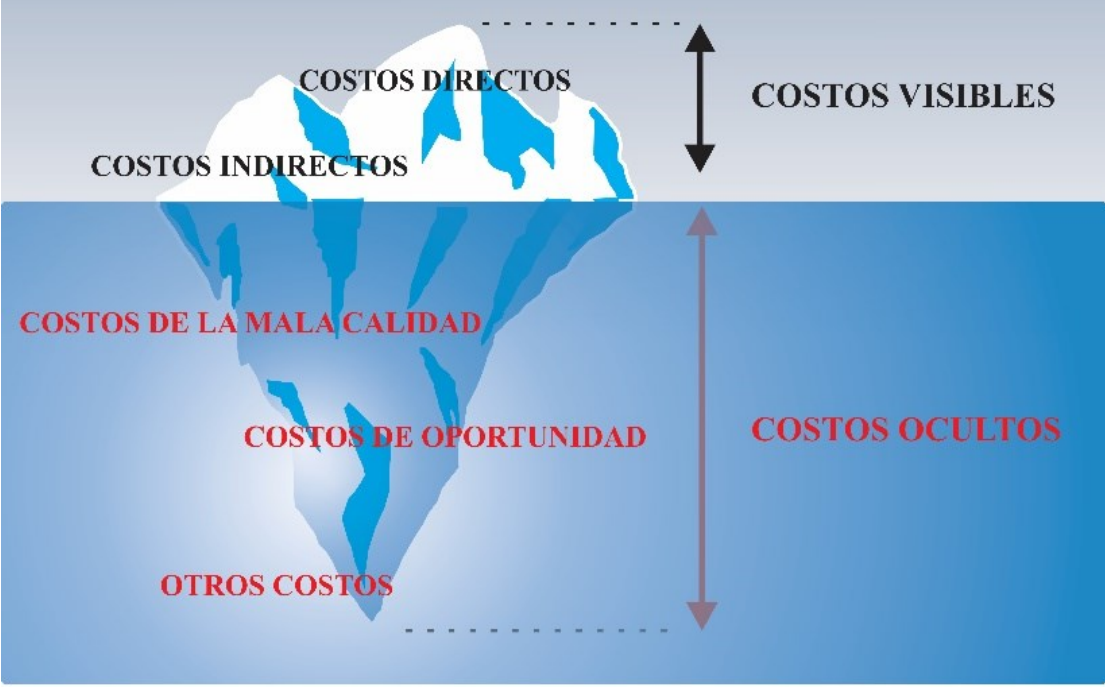

Figura 4. Los costos ocultos de la Cartografía. Tomado y adaptado de $<$ http://www.showeet.com>.

Ejemplos por el mundo de CMC externos relacionados a la Cartografía, López (2013):

- 1998: Alemania, un BMW se precipitó en el río Havel en el embarcadero de un ferry. El conductor seguía fielmente las instrucciones de un sistema GNSS con mapas instalado. El mapa decía "puente" y debió decir "ferry". 
- 1999: Embajada China en Belgrado, bombardeada por la OTAN "por error". Los mapas decían que era una instalación serbia (había sido antes).

- 2010: Costa Rica, "invasión" de Nicaragua a ese país basándose en límites según Google Maps. Un alto mando militar se ampara en la "exactitud" de Google para contravenir toda la cartografía de la historia limítrofe y justificar su incursión y reclamar soberanía.

- 2010: Estados Unidos, Lauren Rosenberg cruzó a pie la autopista y fue atropellada. Creyó lo que le decía Google Maps y siguiendo las instrucciones del GPS cruzó una autopista a pie. Fue atropellada por un auto. En consecuencia demandó a Google Maps por US\$100,000.

- 2013: Filipinas, barreminas encallado. Este barco militar a toda máquina encalla en arrecife de coral. Era un área protegida y existió peligro de derrame de fuel. La carta náutica digital ubicaba el arrecife a 8 millas del lugar.

También hay ejemplos de CMC en Uruguay:

- 1997: Punta del Este, derrame de petróleo de barco petrolero. En pleno verano a $40 \mathrm{~km}$ del centro turístico, por una roca sumergida que no figuraba en las cartas náuticas oficiales, se derramó un estimado entre 200 a 3,000 toneladas.

- 2013: Interconexión eléctrica Brasil-Uruguay.

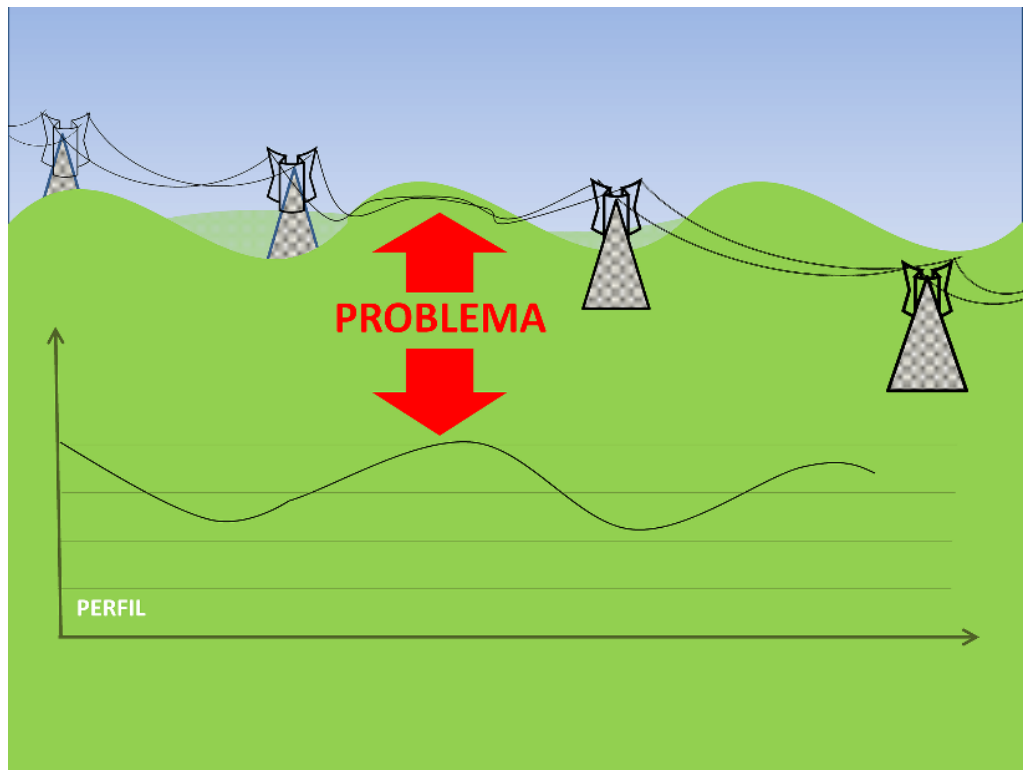

Figura 5. Problema producido por errores en la planialtimetría Fuente: Elaboración propia. 
Dentro del proyecto de interconexión en Extra Alta Tensión entre Brasil y Uruguay, la empresa de energía eléctrica estatal uruguaya tiene que pagar un sobrecosto entre 14,5 y 15,5 millones de dólares a la empresa constructora de la obra, por correcciones en las alturas de 300 torres causadas por "diferencias" con los planos provistos por dicha empresa estatal. El costo primario del proyecto fue de $130 \mathrm{mi}-$ llones de dólares (véase Figura 5).

Este caso en concreto justifica un comentario ampliado ya que los costos incurridos son de público conocimiento. Los planos provistos por la empresa de energía eléctrica fueron realizados por otra empresa privada. Dicho relevamiento planialtimétrico se realizó sobre una faja de $80 \mathrm{~m}$ donde se relevaron cotas del eje de la línea y cotas de los aspectos relativos a la vegetación, uso del suelo, alambrados, caminería, construcciones, cursos de agua, etc., SAAP UTE (2009). La empresa constructora al comenzar el trabajo detecta diferencias entre los planos recibidos y la realidad del terreno. Un estudio realizado por terceros confirma esas diferencias.

El problema más importante fue la necesidad del aumento de tamaño de alrededor de 300 torres. El costo de estas modificaciones fue convenido entre 14,5 y 15,5 millones de dólares, Alem (2013), que la empresa estatal debe pagar.

Hay otras fuentes locales de errores que, de no tenerse en cuenta, pueden resultar en CMC tales como:

- Uso de modelos de elevación defectuosos.

- Uso de origen altimétrico equivocado (tenemos en el país diferentes orígenes aceptados como el Cero Oficial, el Warthon, otros).

- Uso de la zona 21 "ampliada" (zona 22 como si fuera la zona 21) en la proyección UTM sin considerar las anamorfosis (lineal y superficial) que se producen fuera de la zona original. En caso de la superficies éstos pueden ser del orden de $0.2 \%$ del total (por ejemplo, para 500ha (hectáreas) reales nos dará proyectado 1ha más, es decir 501ha).

Simley (2001), menciona fuentes bibliográficas donde expresa que los CMC en la producción cartográfica podrían oscilar entre un $15 \%$ y un $40 \%$ del costo total del producto. Según Climent (2003), en una encuesta realizada en la comunidad valenciana de si se "tienen en cuenta los informes sobre los costes de calidad para la toma de decisiones", la respuesta fue afirmativa en el $90.20 \%$ de los casos. Se puede afirmar que el CMC que muchas veces es despreciado pero que es muy importante y que se debe tener en cuenta en la toma de decisiones. Por eso el CMC para la cartografía se considera un costo oculto. 


\section{Costos y beneficios}

Sin embargo la generación así como el mantenimiento de productos geográficos también es un proceso caro, costoso y complejo. El modelo de análisis de costo beneficio, AGESIC (2013), utiliza para los costos los criterios del TCO (Costo Total de Propiedad), siendo éstos tanto directos como indirectos. Los Costos Directos son los que se pueden identificar o cuantificar plenamente con el producto cartográfico terminado. Ellos serán los sueldos, las tecnologías, el hardware y software, las comunicaciones, las imágenes y fotos aéreas, equipos GNSS, otros materiales, etc. Los Costos Indirectos por el contrario son aquellos que no se pueden identificar o cuantificar plenamente con los productos terminados. Son ejemplos la depreciación de los equipos, sueldos de directores, etc. La publicación de un mapa topográfico nacional a escalas 1:25.000 o 1:50.000 exige realizar todo el proceso cartográfico. Ello implica el concurso de diversos equipos experimentados y bien organizados, una inversión considerable y años de trabajo, Bernabé y López-Vázquez (2012).

Los costos visibles, directos e indirectos, que se podrían estimar para el Uruguay para hacer una nueva cartografía, adaptado de Konecny y otros (2009):

- Cartografía rural (escala 1:50.000 o 1:25.000) de todo el territorio:

Vuelo fotogramétrico, el MDT y la ortofoto $\rightarrow$ US\$ 5 millones y medio

Restitución de los objetos geográficos $\rightarrow$ US\$ 5 millones

Total

$\rightarrow$ US\$ 10 millones y medio

- Cartografía urbana de todos los centros poblados $\left(2.000 \mathrm{~km}^{2}\right)$

Vuelo fotogramétrico, el MDT y la ortofoto $\rightarrow$ US\$ medio millón

Restitución de los objetos geográficos 3D $\rightarrow$ US\$ 14 millones y medio

Total

$\rightarrow$ US\$ 15 millones

Entonces se podría concluir que hacer la cartografía rural del Uruguay podría costar unos 10 millones y medio de dólares. "Sin embargo, si bien las nuevas tecnologías podrían reducir los costos relacionados, los hay y los seguirá habiendo en la creación, gestión y mantenimiento de este contenido", Carpenters y Snell (2013).

Junto a la calidad y los costos, están los beneficios. Existe dificultad para evaluar los beneficios o impactos en una IDE ya que son generalmente intangibles. Estos pueden definirse en términos de eficiencia, eficacia y beneficios socio-políticos (al ciudadano, al gobierno y a los negocios), Workshop (2006). Cuando se habla de eficiencia se refiere al aumento de productividad por ahorro de tiempo, de gastos, por mejores servicios y credibilidad, mayor exactitud y consistencia, etc., algunos tangibles y otros no. Cuando se habla de eficacia se refiere a las políticas y programas que se benefician con mejor información (mejor análisis, formulación de polí- 
ticas, intercambio de información, divulgación, etc.). En resumen se trata de la disponibilidad de recursos y servicios que se ofrecen y cómo la utilizan los usuarios (gobierno, academia, empresas, ciudadanos, etc.). Un resultado esperado es la disminución de la "brecha geomática" entre productores y usuarios de información geográfica. Si se ve desde el punto de vista de gobierno electrónico se puede tomar el modelo para el análisis de los costos y beneficios de, AGESIC (2013) que para los beneficios utiliza dos áreas:

- Para la ciudadanía

- económicos (tiempo, gastos)

- sociales (transparencia, acceso, uso y disponibilidad)

- Para el estado

- económicos (tiempo, gastos)

- sociales (beneficios de gestión tales como gestión humana, calidad de la gestión e imagen)

Por consiguiente, los costos y los beneficios se utilizan para la toma de decisiones en la producción cartográfica. Una forma es cuantificar todos estos beneficios así como los costos incurridos, en una técnica llamada "Análisis Costo-Beneficio", que permite definir si un proyecto es beneficioso o no, brindando un instrumento trascendente para la toma de decisiones. El reto es obtener el financiamiento sustentable para la implantación y operación de estas IDE así como de la obtención y calidad de sus datos, en especial los fundamentales. Para ello será menester mostrarle al Gobierno el valor de los datos así como los beneficios y el impacto que tienen.

Algunos indicadores que se usan:

- VPN o VAN: Valor Presente Neto o Valor Actual Neto

- TIR: Tasa Interna de Retorno (o ROI)

- IVAN: VPN/Inversión

- B/C: Relación Costo-Beneficio

En este ensayo se utiliza el indicador B/C donde el numerador es la cuantificación de todos los beneficios y el denominador de todos los costos incurridos. Si el resultado es $>1$ el proyecto es beneficioso y si es $<1$ no lo es. En el mundo existen muy pocos estudios de este tipo tanto para la IG como para las IDE. Los datos indican valores que oscilan entre 1.82:1 y 23:1, siendo típico un valor de 4:1 (que es lo mismo que expresarlo como 4). Su amplio rango dependía del contexto tecnológico, de casos de uso de aplicación parcial, otras realidades sociales, etc., Workshop (2006). 
En la Tabla 1 se muestran algunos resultados de estudios de Costo/Beneficio:

Tabla 1

\section{Ejemplos de estudios Costo/Beneficios}

\begin{tabular}{|c|c|c|c|c|c|c|c|c|}
\hline \multicolumn{9}{|c|}{ Beneficio Anual } \\
\hline Año & Título & $I D E / I G$ & $B / C$ & $\begin{array}{c}\text { En } \\
\text { mill. } \\
\text { U\$S }\end{array}$ & $\begin{array}{l}\% \text { PBI } \\
\text { de la } \\
\text { región }\end{array}$ & $\begin{array}{l}\% \text { PBI } \\
\text { Región } \\
x \text { PBI } \\
\text { Uruguay } \\
\quad \text { en } \\
\text { mill.U\$S }\end{array}$ & Región & Fuente \\
\hline 2010 & $\begin{array}{l}\text { Valor de la IG } \\
\text { en los servicios } \\
\text { públicos de } \\
\text { Inglaterra y } \\
\text { Gales }\end{array}$ & IG & $2,5: 1$ & 534 & 0,0200 & 11,14 & $\begin{array}{l}\text { Inglaterra } \\
\text { y Gales }\end{array}$ & $\begin{array}{l}\text { Deducido y disponible de: } \\
<\text { http://www.local.gov.uk/c/docu } \\
\text { ment_library/get_file?uuid=b687 } \\
5678-4150-4 d 74-8 b 16- \\
\text { bdd9653f774d\&groupId= } \\
\text { 10180>, accedido el 05-10-2014 }\end{array}$ \\
\hline 2004 & $\begin{array}{l}\text { Un análisis } \\
\text { costo/beneficio } \\
\text { para el Mapa } \\
\text { Nacional de } \\
\text { Estados Unidos }\end{array}$ & IG & $6: 1$ & 120 & 0,0010 & 0,56 & $\begin{array}{l}\text { Estados } \\
\text { Unidos }\end{array}$ & $\begin{array}{l}\text { Deducido y disponible de: } \\
<\text { http://pubs.usgs.gov/circ/2004/ } \\
\text { 1271/>, accedido el 05-10-2014 }\end{array}$ \\
\hline 2011 & $\begin{array}{l}\text { Impacto socio- } \\
\text { económico de } \\
\text { la IDE de } \\
\text { Cataluña (II) }\end{array}$ & IDE & $6,5: 1$ & 2.9 & 0,0012 & 0,67 & Cataluña & $\begin{array}{l}\text { Deducido y disponible de: } \\
<\text { http://www.geoportal- } \\
\text { idec.cat/geoportal/cas/documenta } \\
\text { cio/documents/EstudiImpacteEdi } \\
\text { tatJG_V2_ES.pdf }>\text {, accedido: } \\
\text { 05-10-2014 }\end{array}$ \\
\hline 2004 & $\begin{array}{l}\text { Evaluación de } \\
\text { impacto de } \\
\text { INSPIRE }\end{array}$ & IDE & $8,3: 1$ & 740 & 0,0076 & 4,23 & Europa & $\begin{array}{l}\text { Deducido y disponible de: } \\
<\text { http://www.ec- } \\
\text { gis.org/sdi/ws/costbenefit } 2006 / \text { re } \\
\text { ports/report_sdi_crossbenefit } \% 2 \\
\text { 0.pdf>, accedido el: } 05-10-2014\end{array}$ \\
\hline
\end{tabular}

Fuente: Elaboración propia.

Como se puede apreciar en la Tabla 1 en la tercera columna hay bastante diferencia en los $\mathrm{B} / \mathrm{C}$ anuales y que son como consecuencia de la metodología empleada y de si se refiere a IG o a IDE. En otra columna se presenta el beneficio anual en millones de dólares y el porcentaje de PBI correspondiente a la región del estudio. Luego se proyecta ese porcentaje al PBI de Uruguay y se obtienen valores de 0,56 a 11,14 millones de dólares, que podrían ser los beneficios anuales de la IG o de la IDE en nuestro país. Estos valores son bastante importantes como para despreciarlos aunque podrían estar afectados por la economía de escala. 
Según estudios, CP-IDEA (2011), sólo un 10\% de los países de América participantes de la UN-GGIM Américas, tienen implementado modelos de Retorno de la Inversión y/o monitoreos de los impactos económicos y sociales de las IDE.

\section{Costo de oportunidad}

Sin embargo el análisis Costo-Beneficio puede estar disminuido si no se le complementa con el Costo de Oportunidad. Este costo es el valor de la mejor opción no realizada, Wikipedia (2). Es la renuncia a las oportunidades y posibilidades futuras durante el ciclo de vida del mapa. Los beneficios perdidos al descartar la siguiente mejor alternativa son los costos de oportunidad de la acción escogida. Ese valor normalmente está cargado de subjetividad ya que es muy difícil su medida.

A continuación se va a calcular cuánto hubiera sido el Costo de Oportunidad si no se hubiera tomado la opción de hacer el vuelo fotogramétrico de los años 19661967 en Uruguay. En ese momento se realizaron los vuelos de todo el territorio nacional a dos escalas: 1:40.000 para fines cartográficos y otro al 1:20.000 para atender áreas de suelos, hidrología, catastro, forestación, geología, etc. El vuelo 1:40.000 fue realizado por la empresa Sofratop de Francia. El vuelo a 1:20.000 fue realizado por el Consorcio brasileño Servicios Aerofotogramétricos Vasp, Cruzeiro do Sul, Natividade y Prospec. Ambos vuelos son los únicos de esas características realizados en toda la historia del país. En la Figura 6 se detallan algunos productos cartográficos derivados de esas fotos 1:40.000.

Como se observa en la Figura 6, una profusa producción cartográfica se ha realizado por casi 60 años, hoy ya digitales, derivados de estas fotos. Se muestran en el cuadro más de 40 productos (considerando 1 producto $=1$ plan cartográfico con múltiples hojas), pero hay muchos más, más de 100 productos de diversas instituciones. Esta IG ha permitido el desarrollo de las infraestructuras energéticas, viales, agropecuarias, urbanas, etc., así como atender aspectos sociales, sanitarios, emergencias, de gobierno y administración, entre otros. Se valoriza un sólo producto, la cartografía papel 1:50.000 realizada por el SGM entre 1980 y 1994. Se utiliza el costo de US\$55.000 dado para una carta topográfica 1:25.000 por el USGS en 2007, Craun (2010), aunque sabemos que las nuevas tecnologías han reducido sensiblemente hoy ese valor. Para un total de 300 cartas de nuestro país, estaríamos en 16 millones y medio de dólares de costo del plan. Este sería el Costo de Oportunidad, más algunos millones adicionales al incluir los más de 140 productos derivados, de no haber tomado la CIDE (Comisión de Inversiones y Desarrollo Económico) la decisión de realizar los vuelos fotogramétricos en cuestión. Como este costo muchas veces no se considera en las decisiones, se dice que es un costo oculto. 


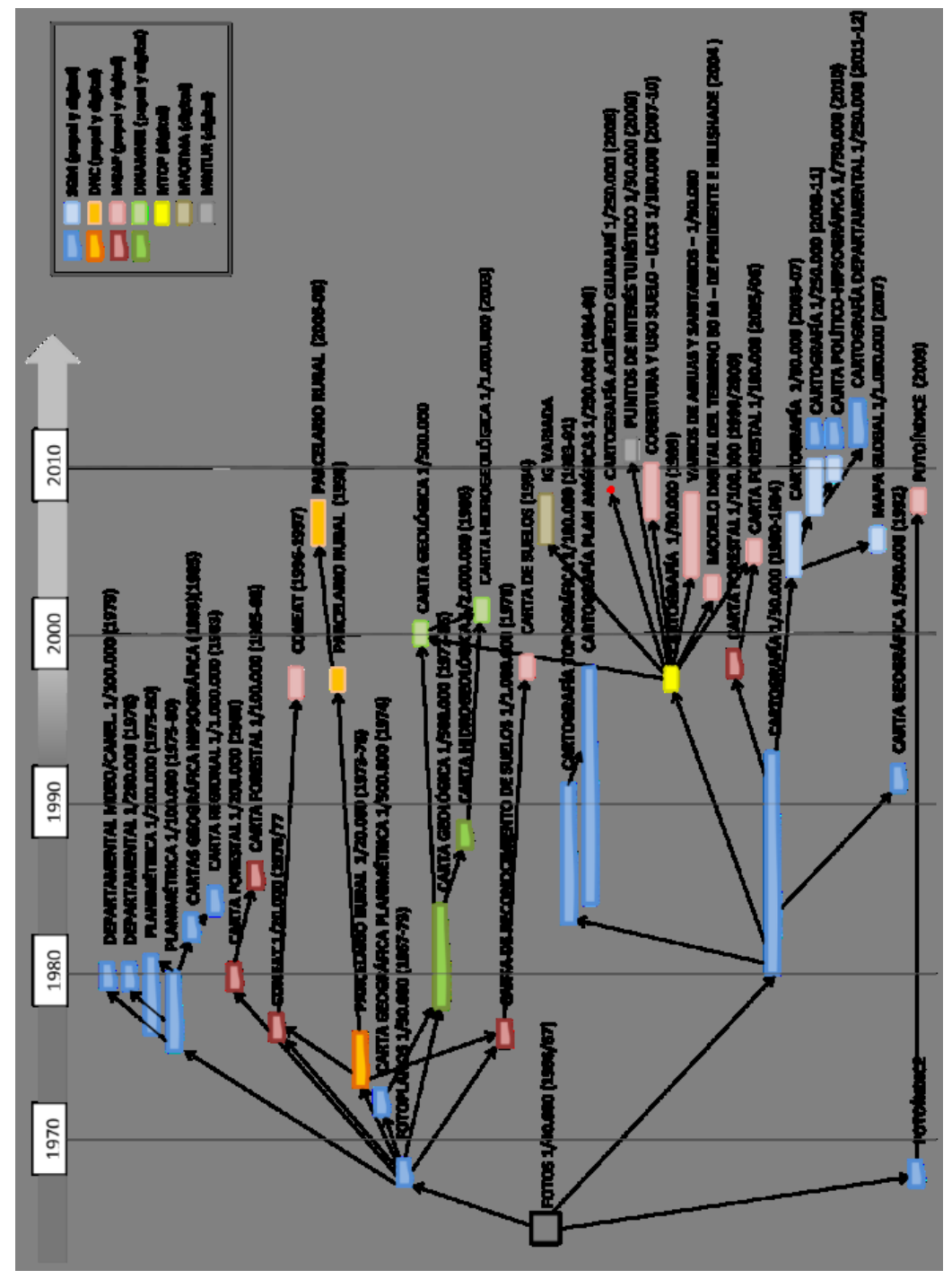

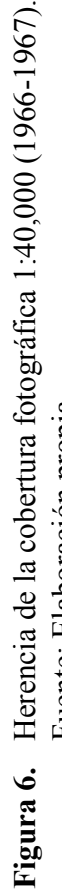




\section{Conclusiones}

Si nuestro país hubiera dispuesto de una cartografía de calidad y oportuna se hubiera evitado el costo de la mala calidad de 15 millones de dólares que el estado debe pagar de sobrecostos. La opción tomada en 1966-1967 de realizar los vuelos fotogramétricos fue la mejor opción y generó beneficios por muchísimo más de 16 millones de dólares. Si no se hubieran realizado esos vuelos el Costo de Oportunidad tomaría ese valor. Entonces, estos "costos ocultos", tomados aislada o conjuntamente con un análisis Costo-Beneficio, pueden y deben, por su magnitud e impacto, ser utilizados para la toma decisiones de inversión en información geográfica.

En conclusión, montos de 5 o 10 millones de dólares para invertir hoy en cartografía parecen más que razonables y oportunos. Podríamos esperar beneficios superiores a 10 o 20 millones de dólares y también evitar los costos de la mala calidad que se están produciendo.

Tendría que definir y asegurarse la sustentabilidad de esta cartografía basándose en ciclos de actualización acordes y financiados.

Hace algunos años, presentando este mismo tema, se comenzó la exposición expresando que la Organización de las Naciones Unidas (ONU) ha dicho que "el desarrollo de un país se mide por el desarrollo de su cartografía" y se finalizó con un proverbio chino que dice "El mejor momento de plantar un árbol fue hace 20 años. El segundo mejor momento es ahora".

¿No será el momento ahora de que tomemos una buena opción?

\section{Bibliografía}

AGESIC (2013), Modelo para el análisis de costos y beneficios, disponible en $<$ http://www.agesic.gub.uy/innovaportal/file/3262/1/modelo_para_el_analisis_d e_costos_y_beneficios.pdf $>$, accedido el 2/10/2014.

Alem (2013), Parlamento Nacional sesión del 27 de noviembre de 2013, Palabras del Gerente de Área de la Asesoría Técnico Jurídica de UTE Dr. Alem, disponible en: <http://www.parlamento.gub.uy/sesiones/AccesoSesiones.asp?Url=/ sesiones/diarios/senado/html/20131127s0060.htm>, accedido el 2/10/2014.

Bernabé, M.A. y López-Vázquez, C. (2012), Fundamentos de las Infraestructuras de Datos Espaciales, UPM Press, Madrid, España, 596 pp.

Canas Torres, J.A. (2001), "La cartografía como infraestructura de las infraestructuras: La Cartografía. Infraestructura para la toma de decisiones", Revista "El campo de las ciencias y las artes.

Carpenters y Snell (2013), Iniciativa de las Naciones Unidas sobre la Gestión Global de la Información Geoespacial, Tendencias a futuro en la gestión de infor- 
mación geoespacial: La visión de cinco a diez años, UN-GGIM (ed.), Naciones Unidas.

Climent (2003), La calidad y su coste como decisiones estratégicas empresariales, Facultad de Economía, Universidad de Valencia, disponible en: < http:// www.uv.es/ $\sim$ scliment/investigacion/2003/seminario.PDF $>$, accedido 2/10/2014.

Clinton, W. (1994). Presidential Documents, Orden Ejecutiva 12906 de creación de la NSDI (EEUU), disponible en: <http://www.archives.gov/federalregister/executive-orders/pdf/12906.pdf>, accedido el 2/10/2014.

CP-IDEA (2011). Diagnóstico sobre temas relevantes de la gestión de información geoespacial y desarrollo de las Infraestructuras de Datos Espaciales - IDE en los países de las Américas, disponible en:

$<$ http://www.cp-idea.org/index.php/component/jdownloads/finish/42-9-reunioncp-idea/245-diagnostico-sobre-temas-relevantes-de-la-gestion-de-informaciongeoespacial-y-desarrollo-de-las-infraestructuras-de-datos-espaciales--ide-en-lospaises-de-las-americas?Itemid=0 $>$, accedido el 12/10/2014.

Craun (2010), Creation of next generation U.S. Geological Survey Topographics Maps, disponible en: <http://www.isprs.org/proceedings/XXXVIII/part4/ files/Craun.pdf>, accedido el 2/10/2014.

FGDC (Federal Geographic Data Committee), 1998, "Geospatial Positioning Accuracy Standards; Part 3: National Standard for Spatial Data Accuracy", FGDCSTD-007.3, Washington, D.C., 28 pp., disponible en: <http://www.fgdc. gov/standards/projects/FGDC-standards-projects/accuracy/part3/chapter3>, accedido el 2014/10/14.

IBM, Big Data, What is big data?, disponible en: <http://www-01.ibm.com/ software/data/bigdata/what-is-big-data.html>, accedido el 2/10/2014.

García M.; Quispe C. y Raez L., (2002). "Costo de la calidad y la mala calidad", Revistas UNMSM, Instituto de Investigación de la Facultad de Ingeniería Industrial, disponible en: <http://sisbib.unmsm.edu.pe/bibVirtual/publicaciones/ indata/v05_n1/calidad.htm>, accedido el 2/10/2014.

Konecny et al. (2009). Economic Considerations for Photogrammetric Mapping, disponible en: <http://gis.vsb.cz/GIS_Ostrava/GIS_Ova_2009/sbornik/Lists/ Papers/067.pdf $>$, accedido el 2/10/2014.

López, C. (2013). Curso de "Control de errores en Datos Geográficos".

López Fojo (2014). Costos de calidad. En busca de la calidad de gestión, Monografía, disponible en: <http://www.monografias.com/trabajos75/costos-calidadcalidad-gestion/costos-calidad-calidad-gestion2.shtml\#ixzz3GbiGUsWx>, accedido el 2/10/2014.

SAAP UTE (2009), Solicitud de Autorización Ambiental Previa, mayo, disponible en: <http://www.ute.com.uy/pags/institucional/documentos/Tomo1Docs/Tomo \%20I_090529_5\%20entrega.pdf >, accedido el 2/10/2014. 
Simley (2001). Cartography and Geographic Information Science, Improving the Quality of Mass Produced Maps.

Wikipedia (1), ISO 8402, disponible en <http://es.wikipedia.org/wiki/ISO_8402>, accedido el 2/10/2014.

Wikipedia (2), Costo de Oportunidad, disponible en <http://es.wikipedia. org/wiki/Coste_de_oportunidad $>$, accedido el 2/10/2014.

Workshop (2006), Reporte del Workshop Internacional sobre Costo-Beneficio / Retorno de la Inversión en las IDEs, disponible en: <http://www.ec_gis.org/sdi// ws/costbenefit2006/reports/report_sdi_crossbenefit\%20.pdf>, accedido el 2/10/ 2014.

\section{Acrónimos}

AGESIC. Agencia para el Desarrollo del Gobierno de Gestión Electrónica y la Sociedad de la Información y del Conocimiento.

B/C. Relación Costo-Beneficio.

CMC. Costos de la Mala Calidad.

CP-IDEA. Comité Permanente para la. Infraestructura de Datos Geoespaciales de las Américas.

DINAMIGE. Dirección Nacional de Minería y Geología.

GNSS. Sistemas de Navegación Global Satelital.

IDE. Infraestructura de Datos Espaciales.

IG. Información Geográfica.

ISO. Organización Internacional de Normalización.

IVAN - VPN. Inversión.

LIDAR. Light Detection and Ranging.

MDT. Modelo Digital del Terreno.

MGAP. Ministerio de Ganadería, Agricultura y Pesca.

MINTUR. Ministerio de Turismo y Deportes.

MTOP. Ministerio de Transporte y Obras Públicas.

MVOTMA. Ministerio de Vivienda, Ordenamiento Territorial y Medio Ambiente.

NSSDA. National Standard of Spatial Data Accuracy.

OTAN.- Organización del Tratado del Atlántico Norte.

PBI. Producto Bruto Interno.

ROI. ReturnOverInvestment - Retorno de la Inversión.

SGM. Servicio Geográfico Militar.

TC 211. Comité Técnico 211 Información Geográfica/Geomática.

TCO. Costo Total de Propiedad.

TIR. Tasa Interna de Retorno.

UN-GGIM Américas. Comité Regional de las Naciones Unidas sobre la Gestión Global de Información Geoespacial para las Américas. 
USGS. U.S. Geological Survey.

VAN. Valor Actual Neto.

VPN. Valor Presente Neto.

Wharton. Es un cero que se ubica a $0,91 \mathrm{~m}$ por debajo del cero oficial. 